This expresses $I_{k, m}(z)$ as a series of functions of the same type. It generalizes the expansion obtained in connexion with the wave functions in Coulomb fields. That expansion corresponds to (5) with $\alpha=0$. In the applications to the wave functions, the parameters $k$ and $z$ are purely imaginary so that it is then convenient to write

and

$$
k=i \eta, \quad z=i \zeta, \quad \alpha=i \lambda
$$

$$
J_{\eta, m}(\zeta)=i^{-(\xi+m)} I_{i \eta, m}(i \zeta)
$$

Making these substitutions in (5), we have

$$
\begin{aligned}
& J_{\eta, m}(\zeta)=\sum_{r=0}^{\infty}(-)^{r} \frac{i^{r+1}(\eta-\lambda) \Gamma\left(i \eta-i \lambda+\frac{1}{2} r\right) \zeta^{\frac{1}{2} r}}{r ! \Gamma\left(i \eta-i \lambda-\frac{1}{2} r+1\right)} J_{\lambda, m+\frac{1}{2} r}(\zeta) \\
& =J_{\lambda, m}(\zeta)+(\eta-\lambda) \zeta^{\ddagger} J_{\lambda, m+\frac{1}{1}}(\zeta)+\frac{1}{2}(\eta-\lambda)^{2} \zeta J_{\lambda, m+1}(\zeta) \\
& +\frac{1}{6}(\eta-\lambda)\left((\eta-\lambda)^{2}+\frac{1}{4}\right) \zeta^{\mathbf{3}} J_{\lambda, m+\frac{1}{1}}(\zeta)+\ldots .
\end{aligned}
$$

The expansions (5) and (7) may be useful in interpolation with respect to the parameters $k$ and $\eta$ for $I_{k, m}(z)$ and $J_{\eta, m}(\zeta)$. It may be noted that, when $k=\eta=0$,

$$
I_{0, m}(z)=\frac{(\pi z)^{\ddagger}}{\Gamma\left(\frac{1}{2}+m\right)} I_{m}\left(\frac{1}{2} z\right), \quad J_{0, m}(\zeta)=\frac{(\pi \zeta)^{\ddagger}}{\Gamma\left(\frac{1}{2}+m\right)} J_{m}\left(\frac{1}{2} \zeta\right)
$$

so that (5) and (7) then yield expansions of the Bessel functions $I_{m}$ and $J_{m}$ in terms of Whittaker's functions.

Faculty of Science,

University of Alexandria, Egypt.

\title{
ON THE GROUP OF A GRAPH WITH RESPECT TO A SUBGRAPH*
}

\section{Frank Harary $\dagger$.}

\section{Introduction.}

A graph $G$ consists of a finite set $P$ of points together with a prescribed collection $L$ of unordered pairs of distinct points called lines. Two points of a graph are adjacent if they belong to a line of the graph. Two graphs $G_{1}, G_{2}$ are isomorphic if there is a one-one mapping of $P_{1}$ onto $P_{2}$ which preserves adjacency. An automorphism of a graph is a selfisomorphism.

* This work was supported by a grant from the National Science Foundation, U.S.A.

† Received 27 November, 1957; read 19 December, 1957; revised 5 March, 1958. 
It is well known (König [6], p. 5) that the set of all automorphisms of a graph $G$ forms a permutation group which acts on $P$. This group is called the group of the graph $G$. We also call it here the point-group of $G$. Our object is to extend this notion to the group $\Gamma(G, H)$ of $G$ with respect to any subgraph $H$ and study some of the consequences of this generalization. Using the powerful enumeration techniques of Pólya [7], we find in the next section a polynomial expression for the number of dissimilar occurrences of collections of copies of a given subgraph $H$ in a graph $G$ in terms of the cyclic structure of $\Gamma(G, H)$. As an application of the method, we obtain a formulation for the number of abstract 2-complexes with a given 1-skeleton. We conclude with some unsolved problems.

Let $\Gamma(G)$ be the group of the graph $G$. By the line group $\Gamma_{1}(G)$ of $G$ we mean the permutation group which acts on the line set $L$ of $G$ which is induced by the elements of $\Gamma(G)$. The group $\Gamma_{1}(G)$ is defined in [5] and in Sabidussi [9] while Tutte [12] essentially defines $\Gamma(G)$ and $\Gamma_{1}(G)$ concurrently. To generalize, the group of a graph $G$ with respect to a subgraph $H$, or more briefly the $H$-group of $G$, is the permutation group $\Gamma(G, H)$ which acts on all subgraphs of $G$ isomorphic to $H$, induced by the automorphisms of $G$. This definition was anticipated in Sabidussi [9], where $M(G, H)$ is defined as the group of all injections of $H$ into $G$. The complete graph $K_{p}$ of $p$ points is the graph in which every two distinct points are adjacent. Let us denote $\Gamma\left(G, K_{p+1}\right)$ by $\Gamma_{p}(G)$. Then $\Gamma_{0}(G)=\Gamma(G)$ and $\Gamma_{1}(G)=\Gamma\left(G, K_{2}\right)$ as above.

\section{Collections of copies of a given subgraph.}

Two points of a graph $G$ are similar if there is an automorphism mapping one onto the other. Similarity of two subgraphs of $G$ is defined analogously. Given a subgraph $H$ of $G$, let $c_{k}$ be the number of dissimilar occurrences in $G$ of $k$ indistinguishable copies of $H$ and let

$$
c(G, H, x)=c_{0}+c_{1} x+c_{2} x^{2}+\ldots .
$$

We wish to derive a formula for this generating function.

We now require Pólya's Theorem [7] in the one-variable form given in [4], p. 447. Since the definitions already appear there, we include only a statement of the theorem here.

Pólya's Theorem. The configuration counting series $F(x)$ is obtained by substituting the figure counting series $\phi(x)$ into the cycle index $Z(\Gamma)$ of the configuration group $\Gamma$. Symbolically:

$$
F(x)=Z(\Gamma, \phi(x)) .
$$

This theorem reduces the problem of finding the configuration counting series $F(x)=c(G, H, x)$ in the present context to the determination of the figure counting series and the cycle index of the configuration group. 
Since any copy of $H$ belonging to $G$ is either absent or present in a collection of copies of $H$, we consider a "set of figures " having just two members, say $\phi_{0}$ and $\phi_{1}$, of weight 0 and 1 respectively. Hence the figure counting series is $\phi(x)=1+x$. The configuration group is clearly $\Gamma(G, H)$. Thus an application of Equation (2) gives the following result.

THEOREM. The generating function for the number of dissimilar occurrences in a graph $G$ of collections of subgraphs isomorphic to $H$ is:

$$
c(G, H, x)=Z(\Gamma(G, H), 1+x) .
$$

Some special cases of this result have already been obtained. One of these is the counting polynomial for all graphs with $p$ points [4], i.e. the polynomial $g_{p}(x)=\Sigma g_{p q} x^{q}$ where $g_{p q}$ is the number of non-isomorphic graphs of $p$ points and $q$ lines :

$$
g_{p}(x)=Z\left(\Gamma_{1}\left(K_{p}\right), 1+x\right)
$$

Another is the polynomial for the number [5] of dissimilar spanning subgraphs of $G$, i.e., subgraphs of $G$ containing all the points of $G$ :

$$
s_{G}(x)=Z\left(\Gamma_{1}(G), 1+x\right)
$$

The exact form of $Z(\Gamma(G, H))$ depends on the particular form of the graphs $G$ and $H$. For certain graph-subgraph combinations, this cycle index has been explicitly formulated.

\section{Example: The number of symmetry types of boolean functions.}

A boolean function of $n$ variables $x_{1}, x_{2}, \ldots, x_{n}$, also sometimes called a switching function in electric network theory, is a function in which the domain of each variable is 0,1 and the range is 0,1 . Two boolean functions $f_{1}$ and $f_{2}$ are of the same type if $f_{1}$ can be transformed into $f_{2}$ by a permutation of the $n$ variables followed by the complementation of a subset (possibly empty) of the variables (the complement of 0 is 1 and of 1 is 0 ).

The 1-skeleton or graph of the $n$-cube, denoted $Q_{n}$, may be defined as the cartesian product of $n$ copies of $K_{2}$, or equivalently as the graph of $2^{n}$ points each of which is a binary sequence of $n$ digits such that two points are adjacent whenever they differ in exactly one digit. It has been shown by Pólya $([8], 102)$ that there is a one-one correspondence between the boolean functions of $n$ variables and the subsets of the set of points of $Q_{n}$ such that two functions are of the same type if and only if their corresponding subsets of points are similar in $Q_{n}$. Hence the result of Pólya [8] that the counting polynomial for the symmetry types of boolean functions of $n$ variables is given by $Z\left(\Gamma\left(Q_{n}\right), 1+x\right)$ is an application of a special case of the Theorem (3). A detailed algorithm for finding the cycle index $Z\left(\Gamma\left(Q_{n}\right)\right)$ has been given by Slepian [11]. 
The polynomial $Z\left(\Gamma\left(Q_{n}, Q_{m}\right), 1+x\right)$ gives for all values of $k$ the number of dissimilar sets of $k m$-cubes in $Q_{n}$. The composition of two permutation groups $A$ and $B$, introduced by Pólya [7] under the name "Gruppenkranz" and denoted $A[B]$, provides the mechanism for expressing one class of these polynomials, namely the case $m=n-1$, in closed form. This operation is also discussed in Frucht [3]. For it is easily seen (cf. Pólya [8], footnote 7) that $\Gamma\left(Q_{n}, Q_{n-1}\right)=S_{n}\left[S_{2}\right]$, the automorphism group of the hyperoctahedron. Hence the corresponding counting polynomial is

$$
c\left(Q_{n}, Q_{n-1}, x\right)=Z\left(S_{n}\left[S_{2}\right], 1+x\right) .
$$

It is shown in Pólya [7], p. 180, that for any permutation groups $A$ and $B$, $Z(A[B])$ is the composition of $Z(A)$ with $Z(B)$. This, together with the usual formula for $Z\left(S_{n}\right)$ gives this polynomial concisely, in the form:

$$
c\left(Q_{n}, Q_{n-1}, x\right)=\sum_{(j)}^{n} \prod_{i=1}^{n} \frac{\left(1+x^{i}+x^{2 i}\right)^{j_{i}}}{i^{j_{i}} j_{i} !},
$$

where the sum is taken over all partitions $(j)$ of $n$, i.e., all $n$-tuples $(j)=\left(j_{1}, j_{2}, \ldots, j_{n}\right)$ such that

$$
1 j_{1}+2 j_{2}+\ldots+n j_{n}=n \text {. }
$$

4. The number of 2-complexes with given 1-skieleton.

One of the unsolved problems stated in [4] is to enumerate the nonisomorphic abstract simplicial complexes with a given number of simplexes of each dimension. An application of the theorem yields partial information by supplying an enumeration for non-isomorphic 2-complexes with prescribed 1-skeleton $G$. Let $c_{2}(G, r)$ be the number of isomorphism classes (defined in the usual way) of 2-complexes whose 1-skeleton is $G$, containing $r(\geqslant 0)$ 2-simplexes. Let $c_{2}(G, x)=\underset{r}{\sum_{2}} c_{2}(G, r) x^{r}$. Then by the theorem, we immediately have

$$
c_{2}(G, x)=Z\left(\Gamma\left(G, K_{3}\right), 1+x\right) .
$$

For example, if $G$ is the graph with six points consisting of an equilateral triangle and the triangle determined by the midpoints of its sides, then $\Gamma\left(G, K_{3}\right)=S_{3} \cdot S_{1}$ (direct product) so that

Thus

$$
Z\left(\Gamma\left(G, K_{3}\right)\right)=\frac{1}{6}\left(f_{1}^{4}+3 f_{1}^{2} f_{2}+2 f_{1} f_{3}\right) .
$$

$$
\begin{aligned}
c_{2}(G, x) & =\frac{1}{6}\left[(1+x)^{4}+3(1+x)^{2}\left(1+x^{2}\right)+2(1+x)\left(1+x^{3}\right)\right] \\
& =1+2 x+2 x^{2}+2 x^{3}+x^{4} .
\end{aligned}
$$

Diagrams which verify this last polynomial are readily drawn. 


\section{Problem.}

It was first shown, constructively, by Frucht [1] that for any given abstract group, there exists a graph whose group is isomorphic to the given group. This result was subsequently extended by Frucht [2] to cubical graphs with a given abstract group and more recently by Sabidussi [10] to graphs with given group and given graph-theoretic properties. However, none of these results is directed toward the question of which permutation groups belong to a graph. It is known that not all permutation groups have this property. For example any cyclic group of degree $n$ and order $n$ for $n>2$ does not belong to any graph. The approach of the present paper raises similar questions. For the sequence of permutation groups $\Gamma_{0}(G), \Gamma_{1}(G), \Gamma_{2}(G), \ldots$ is an invariant of the graph $G$. To what extent is this sequence a complete set of invariants for $G$ ? Given a sequence of abstract groups $A_{0}, A_{1}, A_{2}, \ldots$, under what conditions does there exist a graph $G$ such that for all $n, \Gamma_{n}(G)$ is abstractly isomorphic to $A_{n}$ ? In particular, given an abstract group $A_{0}$, which groups $A_{1}$ have the property that the pair $\left(A_{0}, A_{1}\right)$ are isomorphic to the point-group and line-group of the same graph?

\section{References.}

1. R. Frucht, "Herstellung von Graphen mit vorgegebener abstrakter Gruppe ", Compositio Math, 6 (1938), 239 250.

2. - - - "Graphs of degree three with a given abstract group ", Canadian J. of Math., 1 (1949), 365-378.

3. _-__, "On the groups of repeated graphs", Bull. American Math. Soc., 55 (1949), $418-420$.

4. F. Harary, "The number of linear, directed, rooted and connected graphs", Trans. American Math. Soc., 78 (1955), 445-463.

5. - - " On the number of dissimilar line-subgraphs of a given graph ", Pacific $J$. of Math., 6 (1956), 57-64.

6. D. König, Theorie der endlichen und unendlichen Graphen (Leipzig, 1936; reprinted New York, 1950).

7. G. Pólya, "Kombinatorische Anzahlbestimmungen für Gruppen, Graphen, und chemische Verbindungen ", Acta Math., 68 (1937), 145-254.

8. ___- "Sur les types des propositions composées", J. Symbolic Logic, 5 (1940), 98-103.

9. G. Sabidussi, "Loewy-groupoids related to linear graphs ", American $J$. of Math., 76 (1954), 477-487.

10. - - " "Graphs with given group and given group-theoretic properties ", Canadian $J$. of Math., 9 (1957), 515-525.

11. D. Slepian, "On the number of symmetry types of Boolean functions of $n$ variables ", Canadian $J$. of Math., 5 (1953), 185-193.

12. W. T. Tutte, "A family of cubical graphs", Proc. Cambridge Phil. Soc., 43 (1948), $459-474$.

University of Michigan and

The Institute for Advanced Study. 